\title{
Association of Serum $\beta$-Hydroxybutyrate and Coronary Artery Disease in an Urban Chinese Population
}

Hongna Mu

Beijing Hospital

Ruiyue Yang

Beijing Hospital

Siming Wang

Beijing Hospital

Wenduo Zhang

Beijing Hospital

Xinyue Wang

Beijing Hospital

Hongxia Li

Beijing Hospital

Jun Dong

Beijing Hospital

Wenxiang Chen

Beijing Hospital

Xue Yu

Beijing Hospital

Fusui Ji ( $\sim$ ji_fusui@163.com )

Beijing Hospital https://orcid.org/0000-0002-0068-9472

\section{Research}

Keywords: Coronary artery disease, ketone bodies, $\beta$-hydroxybutyrate, Gensini score

Posted Date: November 22nd, 2021

DOI: https://doi.org/10.21203/rs.3.rs-1029455/v1

License: (1) (1) This work is licensed under a Creative Commons Attribution 4.0 International License.

Read Full License 
Version of Record: A version of this preprint was published at Frontiers in Nutrition on February 17th, 2022. See the published version at https://doi.org/10.3389/fnut.2022.828824. 


\section{Abstract}

Background: Ketone bodies, including $\beta$-hydroxybutyrate, acetoacetate, and acetone, can substitute and alternate with glucose under conditions of fuel and food deficiency. Ketone body metabolism is increased in a myriad of tissue metabolism disorders. Perturbations in metabolism are major contributors to coronary artery disease (CAD). The present study aimed to investigate the association of $\beta$ hydroxybutyrate with CAD.

Methods: A total of 2970 Chinese Han population were enrolled. Gensini scores were calculated for all patients with positive findings. Serum levels of $\beta$-hydroxybutyrate and other laboratory parameters were measured. Associations of serum metabolites with traditionary risk factors and CAD severity were analyzed.

Results: Our results showed that $\beta$-hydroxybutyrate was associated with some CAD risk factors and CAD severity, determined by Gensini score or number of diseased regions. Moreover, $\beta$-hydroxybutyrate was associated with $\mathrm{T} 3 / \mathrm{T} 1$ of Gensini score tertile after adjusting for traditional risk factors by multivariable logistic regression analysis. The association of $\beta$-hydroxybutyrate with CAD severity was more obvious in female.

Conclusions: Taken together, circulating $\beta$-hydroxybutyrate level was independently associated with CAD severity, and the association was more pronounced in female.

\section{Introduction}

Coronary artery disease (CAD), caused by atherosclerosis, has been reported as the leading cause of death worldwide. Although new medical and therapies have emerged in the last years, the risk of deaths in the course of CAD is still high[1]. Therefore, there is a need for the development of better diagnostic methods, novel biomarkers, more effective drugs as well as new therapeutic targets, which can contribute a lot to the decrease of CAD mortality[2, 3].

Metabolic dysfunction is a hallmark of CAD pathophysiology, reflecting not only the altered metabolism of the myocardium but rather overall contributions from peripheral tissues and organs[4,5]. These metabolic changes directly aids in the disease pathophysiology and may serve as earlier and more sensitive markers of CAD [6, 7]. Abnormal accumulation or deficiency of specific metabolites within the circulation can be powerful markers in the management of CAD, with regard to detecting disease, evaluating disease progression, and assessing therapeutic effectiveness[8, 9].

$\beta$-hydroxybutyrate ( $\beta$-HDBA) is a major component of ketone body, which derives from increased free fatty acid beta oxidation[10]. When perturbed tissue metabolism happens, an increase in $\beta$-HDBA (ketone bodies) may help circumvent the metabolic malfunctions that directly contributes to disease pathophysiology[11]. Recent studies have showed that $\beta$-HDBA level was increased in a myriad of pathological conditions, including type 2 diabetes[12], arrhythmogenic cardiomyopathy[13] and heart 
failure[14]. In addition, it was reported that $\beta$-HDBA was elevated in non-ST segment elevation acute coronary syndrome patients, reflecting the oxidative stress and the hypoxic state that suffers the myocardial cells, which indicated that $\beta$-HDBA may have a great potential for early diagnosis of acute coronary syndrome[15]. Another report has shown that increased serum $\beta$-HDBA level was independently associated with cardiovascular events and all-cause death in hemodialysis patients[16]. The above studies implied potential association of $\beta$-HDBA with CAD. However, to our knowledge, no studies have examined the associations between $\beta$-HDBA and CAD in Chinese Han populations. Here, we evaluated the associations of $\beta$-HDBA with CAD, severity of CAD, and CAD risk factors in a case-control study.

\section{Materials And Methods \\ 2.1 Study population}

This is a cross-sectional study approved by the Ethics Committee of Beijing Hospital (2016BJYYEC-12103) and all subjects provided written informed consent. Patients who were suspected CAD or adjudicated CAD history and subjected to coronary angiography in Beijing Hospital between March 2017 and October 2020 were enrolled. We specifically excluded patients with severe congenital heart disease, severe cardiac insufficiency, primary pulmonary hypertension, hepatic and renal dysfunction, and severe peripheral arterial disease or related conditions which are contraindications of cardiac catheterization; patients who was receiving radiotherapy or chemotherapy, pregnant or nursing, alcoholism or drug abuse, and patients with mental illness under treatment. Therefore, 2970 consecutive patients were included in this study. The subjects' demographics (eg, age and sex), medical history (eg, diabetes mellitus, hypertension, and dyslipidemia), cigarette smoking, and body mass index (BMI) were recorded. Before the coronary angiography procedure, venous blood samples were collected after fasting overnight, serum was isolated, aliquoted and stored at $-80^{\circ} \mathrm{C}$ until analyses.

\subsection{Coronary angiography}

Coronary angiography was performed for CAD assessment by experienced cardiologists. All targeted coronary lesions of enrolled patients were analyzed by the built-in QCA software of the Allura Xper FD20 Angiography System (Philips Healthcare, Netherlands), and all coronary segments were analyzed quantitatively using a 15-segment American Heart Association classification. According to the current guidelines, coronary stenosis was defined as a decrease of more than $50 \%$ in the arterial diameter, and if not, was diagnosed as non CAD. In addition, individuals who reported with negative findings in computed tomography of the coronary arteries or stress myocardial perfusion imaging were also categorized as the non CAD group.

\subsection{Gensini score}

The Gensini score was used to assess the severity of coronary artery damage[17]. Gensini score was computed by assigning a severity score for each coronary stenosis depending on the degree of luminal narrowing and its importance based on location. Luminal stenoses of $25 \%, 50 \%, 75 \%, 90 \%, 99 \%$, and total 
occlusion were given Gensini score of $1,2,4,8,16$, and 32, respectively. These scores were then multiplied by a factor according to location: 5 , left main coronary artery; 2.5 , proximal segment of the left anterior descending coronary artery and proximal segment of the circumflex artery; 1.5 , mid-segment of the left anterior descending coronary artery; 1.0 , right coronary artery, distal segment of the left anterior descending coronary artery, posterior descending artery, and obtuse marginal artery; and 0.5 , other segments. Finally, the Gensini score was calculated by summation of the individual coronary segment scores. The Gensini score was calculated in 2047 patients who did not have previous percutaneous artery interventions.

\subsection{LC-MS/MS measurement for $\beta$-HDBA}

The serum concentration of $\beta$-HDBA was quantified using an LC-MS/MS system consisted of a 1260 Infinity II Series HPLC (Agilent, Santa Clara, CA, USA) coupled with a Sciex 5500 QTRAP mass spectrometer ( $A B$ Sciex, Foster City, CA, USA). The detailed mass spectrometry parameters were set as previously described[18]. $\beta$-HDBA standards, serum samples and quality controls were respectively mixed with the isotopically labeled internal standard ( $\beta$-HDBA-D4) solution and proteins were precipitated with methanol containing $0.5 \%$ formic acid. After vortexing and centrifuging, the metabolites were separated by a Kinetex ${ }^{\mathrm{TM}}$ hydrophilic interaction LC (HILIC) column $(2.6 \mu \mathrm{m}, 150 \times 2.1 \mathrm{~mm}$, Phenomenex, Torrance, CA, USA) and eluted with a mobile phase of $75 \%$ methanol, containing $5 \mathrm{mmol} / \mathrm{L}$ ammonium formate and $0.05 \%$ formic acid at a flow rate of $0.3 \mathrm{~mL} / \mathrm{min}$. $\beta$-HDBA and internal standard were detected with positive electrospary ionization (ESI) in multiple reaction monitor (MRM) mode using characteristic precursor-product ion transitions of $m / z 103.0 \rightarrow 59.0$ and $m / z 107.0 \rightarrow 59.0$, respectively. The concentration of $\beta$-HDBA was calculated by standard curves. The analytical coefficients of variation for $\beta$ HDBA measurements was below 3\%. Applied Biosystems Analyst version 1.6.2 software was used for system control, data collection, and processing.

\subsection{Other parameters and laboratory testing}

The serum samples were also tested for fasting blood glucose (FBG), total cholesterol (TC), triglycerides (TG), high density lipoprotein cholesterol (HDL-C), low density lipoprotein cholesterol (LDL-C), creatinine (Crea) and uric acid (UA) using assay kits (Sekisui Medical Technologies, Osaka, Japan) on a Hitachi 7180 chemistry analyzer. Two quality control materials, prepared by mixed fresh serum samples, were analyzed with patient samples in each run in both the LC-MS/MS method and the laboratory assays to monitor the performance of the measurements.

\subsection{Statistical analysis}

All variables were tested for normality. Parameters with normal distributions were expressed as means \pm SD, while parameters with skewed distributions were presented as medians and percentile (25th to 75th percentile). Count data were reported as frequencies and percentages. The one-way analysis of variance test was conducted to compute the differences for continuous variables, while the nonparametric Jonckheere-Terpstra test was used to determine the differences when the data were not normally distributed. For categorical variables, the Chi-square test was used. Spearman correlation analysis was 
used to examine the associations between $\beta$-HDBA and traditional CAD risk factors. The multivariable logistic regression analysis was used to determine the relation of $\beta$-HDBA level with Gensini score. Potential confounding variables, including age, gender, smoking status, obesity or overweight, hypertension, dyslipidemia, diabetes, history of stroke and family history of premature CAD, were controlled in the regression models. Results were presented as odds ratios (OR) and $95 \%$ confidence intervals $(95 \% \mathrm{Cl})$. The reported $P$-values were two-tailed, with $P$-values $<0.05$ considered statistically significant. Statistical analysis was performed using IBM SPSS Statistics v. 25.0 software (IBM Corp., Armonk, NY, USA).

\section{Results}

\section{Clinical Characteristics}

The study population comprised a total of 2970 patients in which $62.5 \%$ were males. The baseline characteristics according to tertile of $\beta$-HDBA levels were summarized in Table 1 . The results showed that subjects with higher $\beta$-HDBA levels also had higher SBP, higher prevalence of diabetes, dyslipidemia and family history of premature CAD. Furthermore, with the increase of $\beta$-HDBA levels, FBG, TC and LDL-C significantly increased $(P<0.001$ for trend). It seems that subjects with higher $\beta$-HDBA levels were older, but it is not statistically significant. Whereas BMI, DBP, TG, HDL-C, Crea and UA levels were similar among the groups. In addition, no associations were found between $\beta$-HDBA and overweight/obesity, hypertension, history of stroke, smoking status and statins use.

\section{Correlations of serum $\beta$-HDBA levels with the traditional CAD risk factors}

Significant positive correlations were found between serum $\beta$-HDBA level and FBG $(\mathrm{r}=0.067, P<0.001)$, TC $(\mathrm{r}=0.070, P<0.001)$, and LDL-C ( $\mathrm{r}=0.066, P=0.001)$. Serum $\beta$-HDBA level was also significantly positively correlated with age $(r=0.041, P<0.05)$ and SBP $(r=0.040, P<0.05)$. However, associations between $\beta$-HDBA and BMI, DBP, TG, HDL-C, Crea and UA were not significant (Table 2).

\section{Relations of $\beta$-HDBA levels with CAD severity}

In order to analyze the relationships between $\beta$-HDBA and CAD severity, patients with positive findings in coronary angiography were classified into subgroups with 1-3 and $>3$ stenosed regions. $\beta$-HDBA was significantly increased $\left(P_{\text {trend }}=0.016\right)$ with the increase of the number of stenosed regions. Then the CAD patients were further divided into subgroups with 1 and $>1$ stenosed vessles. As shown in Fig 1, no significant changes were found for $\beta$-HDBA levels among groups. As the Gensini score is widely used for evaluating of coronary atherosclerosis severity, the studied patients were further divided into different subgroups according to tertiles of the Gensini score. The results indicated that $\beta$-HDBA level was found to be associated with the Gensini scores $\left(P_{\text {trend }}=0.05\right)$.

\section{The multivariable logistic regression analysis of $\beta-H D B A$ level with CAD}


To examine the independent relationship between $\beta$-HDBA and CAD severity, multivariate logistic regression analysis was performed. Here, we adopted T3/T1 of Gensini score tertiles on behalf of CAD severity. As shown in Table 3, traditional risk factors such as age, gender, smoking (both former and current smoking), hypertension, diabetes, family history of premature CAD were all positively associated with the Gensini scores, while in gender, female was a protective factor for CAD, with OR of 0.417 (0.3080.564). In addition, we found elevated $\beta$-HDBA levels remained independently associated with $T 3 / T 1$ of Gensini score tertiles (OR=1.151, 95\% confidence interval:1.012-1.309, $p=0.032$ ), after adjusting for traditional risk factors including age, gender, smoking status, overweight/obesity, hypertension, dyslipidemia, diabetes, history of stroke, family history of premature CAD.

\section{The stratified analysis of $\beta$-HDBA levels with CAD}

Stratified analysis of the associations of serum $\beta$-HDBA levels (per 1-SD increment) and the severity of coronary artery lesion was conducted in different groups of age, gender, BMI, smoking status, hypertension, dyslipidemia, diabetes, history of stroke and family history of premature CAD. The interactions between $\beta$-HDBA and these factors on the distribution of CAD were analyzed. As presented in Fig. 1, there are significant associations between $\beta$-HDBA and CAD in female, non-smokers, subjects with hypertension, diabetes and family history of premature CAD. Moreover, we observed that the association between $\beta$-HDBA and CAD was modified by gender $\left(P_{\text {interaction }}=0.015\right)$. $\beta$-HDBA was associated with CAD in female (HR: $1.429,95 \% \mathrm{Cl}: 1.138-1.793, P=0.002)$, but not in male (HR: $1.028,95 \% \mathrm{Cl}: 0.892-1.1840, P$ $=0.704$ ), after adjustment for age, BMI, smoking status, hypertension, dyslipidemia, diabetes, history of stroke, family history of premature CAD. However, no interaction was observed between $\beta$-HDBA and age, $\mathrm{BMI}$, smoking status, hypertension, dyslipidemia, diabetes, history of stroke and family history of premature CAD (Figure 2). Then we further analyzed the association of $\beta$-HDBA level with CAD severity in male and female respectively, according to Gensini score or the number of stenosed regions. Consistent with this, $\beta$-HDBA showed significant association with Gensini score or the number of stenosed regions in female, not male (Figure 3).

\section{Discussion}

In this study, we observed circulating $\beta$-HDBA concentration was associated with CAD traditional risk factors and CAD severity. An elevated $\beta$-HDBA level was associated with an increased CAD severity degree, defined by Gensini score or number of stenosed regions. This association of $\beta$-HDBA level with Gensini score remained significant after adjusting for conventional CAD risk factors by multivariable logistic regression analysis. Increased levels of $\beta$-HDBA in CAD patients indicated a more severe state of the disease and more severe systemic metabolic perturbations, especially in female. To the best of our knowledge, this study is the first to report that an elevated $\beta$-HDBA level is associated with an increased risk of CAD in Chinese population.

As heart is the most metabolically demanding organ in the body, impaired metabolism may lead to CAD, thus, identify and quantify changes in these metabolites is critical for diagnosis and treatment of 
CAD[19]. Ketone bodies, including $\beta$-HDBA, acetoacetate, and acetone, are small fuel substrates, derived from increased free fatty acid beta oxidation. These substances can uniquely substitute and alternate with glucose under conditions of fuel and food deficiency[20]. There is by now plenty of evidence that ketone bodies, in particular $\beta$-HDBA, elevated in metabolic disorders. The most robust evidence from a retrospective cohort study demonstrated that $\beta$-HDBA was independently and significantly associated with adverse cardiovascular events and therefore could serve as a novel biomarker predicting both survival and incidents of cardiovascular events in patients with hemodialysis[16]. Accumulating evidence has also shown that $\beta$-HDBA levels were elevated in patients with heart failure[14, 21], obese and/or type 2 diabetes[12, 22]. Of importance, with metabolic dysfunction as an inherent feature of the CAD pathophysiology, whether circulating $\beta$-HDBA is increased in CAD remains poorly understood. Given above promising findings, we explored a study to examine whether $\beta$-HDBA is associated with incident CAD in Chinese population.

In this study, we first analyzed the association of circulating $\beta-H D B A$ concentration with CAD traditional risk factors. The result revealed strong associations of $\beta$-HDBA with FBG,TC and LDL-C, as well as significant association between $\beta$-HDBA and age and SBP. These results were not in line with some of the previous reports[13,23], possibly on account of different population (Table 2).

Then we analyzed the relation of $\beta$-HDBA with CAD and CAD severity. The study population was first divided into non-CAD group and CAD group according to coronary angiography. Beyond expectation, subjects who developed CAD did not have significantly higher $\beta$-HDBA level in serum compared with controls (data not shown). While in a Japanese pilot study, the authors found $\beta$-HDBA was significantly lower in the CAD group compared with control subjects in patients with type 2 diabetes. However, the number of CAD subjects and controls are quite small in this study[5]. To analyze the associations between $\beta$-HDBA and CAD severity, the CAD group was further divided into subgroups, based on the number of stenosed vessles or regions. The results revealed that $\beta$-HDBA was associated with the severity of CAD grouped by the number of stenosed regions. Gensini score is widely used for evaluating coronary atherosclerotic severity with judgement of the position, number and stenosis of blood vessels caused by coronary atherosclerosis[17]. The CAD patients were grouped into 3 groups according to Gensini score tertile, and $\beta$-HDBA showed significant association with Gensini score tertile (Figure 1). In multivariable logistic regression analysis, $\beta$-HDBA was independently associated with T3/T1 of Gensini score tertile CAD, even after adjusting for other well-known risk factors (Table 3).

By further stratification analysis, it turned out that $\beta$-HDBA displayed good association with CAD in female (Figure 2). Furthermore, in accordance with this, Figure 3 revealed that $\beta$-HDBA level was associated with CAD severity in female not male, by the standard of Gensini score or the number of diseased regions. These results indicated that $\beta$-HDBA may significantly contribute to the progress of atherosclerosis especially in female. Actually, cardiac metabolism and risk factor burden differed by sex[24, 25], sex may have an influence on both the development and progression of CAD and on the pattern of compositional plaque progression, and female have higher mortality rates[26]. Thus these 
messages suggested that $\beta$-HDBA may remind of CAD state in female, and we will pay attention to the role of $\beta$-HDBA played in CAD development and progress in female population.

Traditionally, severe ketosis, or ketoacidosis, is known as a harmful event. Ketone bodies are synthesized in liver from acetyl-CoA derived primarily from fatty acid $\beta$-oxidation and are transported to extrahepatic tissues for terminal oxidation as a source for ATP generation at the expense of glucose oxidation[27]. Growing evidence indicated that the failing heart primarily consumed fatty acids and, unexpectedly, little glucose[28, 29]. Myocardial ischemia occurs when coronary blood flow is inadequate, and hence, the oxygen supply to the myocardium is not sufficient to meet oxygen demand. Ischemia elicits disturbances in the balance between energy metabolism and cardiac function[30]. It seems that hepatic ketogenesis was activated and cardiac utilization was not enhanced, the concentration of blood ketone bodies was highly increased in ischemic heart disease patients[24,30]. A study in humans also support this concept, CAD patients exhibited deficits metabolic fuel extraction, with reduced baseline fatty acid and ketone extraction compared to controls[31]. However, recently evidence from both experimental and clinical research has uncovered a protective role for ketones in cardiovascular disease. Indeed, ketone bodies possess a variety of capabilities and participate in multiple cellular processes[32]. $\beta$-HDBA infusion specifically improved working memory performance in patients with type 2 diabetes in the absence of changes in global cognition[33]. In addition, the data from a study in mice unearthed that chronic elevation of circulating $\beta$-HDBA protected against the development of heart failure that is associated with the ability of $\beta$-HDBA to directly reduce inflammation[34]. Furthermore, $\beta$-HDBA was found to protect heart from ischemia/reperfusion injury[35] and attenuate atherosclerosis[36] in different mice models. Cumulatively, further studies to prove the action and specific mechanism of $\beta$-HDBA in CAD should be warranted.

\section{Conclusion}

In this study, we found circulating $\beta$-HDBA was independently associated with CAD severity, and further elucidated the gender-related difference in this association. Further studies are necessary to determine the specific role of $\beta$-HDBA in CAD.

\section{Abbreviations}

$\beta$-HDBA, $\beta$-hydroxybutyrate; $B M I$, body mass index; CAD, coronary artery disease; Crea, creatinine; DBP, diastolic blood pressure; FBG, fasting blood glucose; HDL-C, high density lipoprotein cholesterol; LDL-C, low density lipoprotein cholesterol; SBP, systolic blood pressure; TC, total cholesterol; TG, triglyceride; UA, uric acid;

\section{Declarations}

\section{Ethics approval and consent to participate}


This study was approved by the Ethics Committee of Beijing Hospital (2016BJYYEC-121-03) and all subjects provided written informed consent.

\section{Consent for publication}

Not applicable.

\section{Availability of data and materials}

The datasets used and/or analysed during the current study are available from the corresponding author on reasonable request.

\section{Competing interests}

The authors declare that they have no conflicts of interest.

\section{Funding}

This work was supported by Beijing Natural Science Foundation (grant number 7214250), the National Key R\&D Program of China (2020YFC2008304), the Non-profit Central Research Institute Fund of the Chinese Academy of Medical Sciences (grant number 2019TX310001) and Beijing Hospital Special Fund Project (grant number BJ-2019-184).

\section{Authors' Contributions}

HN M: Conceptualization, Methodology, Writing- Original draft preparation. RY Y: Data curation, Validation, Formal analysis. SM W: Methodology, Data curation, Formal analysis. WD Z: Methodology, Data curation. XY W: Data curation, Formal analysis. HX L: Formal analysis. J D: Data curation, Project administration. WX C: Project administration. X Y: Conceptualization, Writing-Reviewing and Editing. FS $\mathrm{J}$ : Conceptualization, Writing-Reviewing and Editing, Funding acquisition, Project administration. All authors read and approved the final manuscript.

\section{Acknowledgements}

The authors thank Ranran Zhang and Ziyun Li for excellent technical assistance.

\section{Authors' information}

${ }^{1}$ The Key Laboratory of Geriatrics, Beijing Institute of Geriatrics, Institute of Geriatric Medicine, Chinese Academy of Medical Sciences, Beijing Hospital/National Center of Gerontology of National Health Commission, P.R. China

${ }^{2}$ Department of Cardiology, Beijing Hospital, National Center of Gerontology, Beijing, 100730, P. R. China

${ }^{3}$ National Center for Clinical Laboratories, Beijing Hospital, National Center of Gerontology; Institute of Geriatric Medicine, Chinese Academy of Medical Sciences, P.R. China 


\section{References}

1. Senthong V, Li XS, Hudec T, Coughlin J, Wu Y, Levison B, Wang Z, Hazen SL, Tang WH. Plasma Trimethylamine N-Oxide, a Gut Microbe-Generated Phosphatidylcholine Metabolite, Is Associated With Atherosclerotic Burden. J Am Coll Cardiol. 2016;67:2620-8.

2. Tunon J, Barbas C, Blanco-Colio L, Burillo E, Lorenzo O, Martin-Ventura JL, Mas S, Ruperez FJ, Egido J. Proteomics and metabolomics in biomarker discovery for cardiovascular diseases: progress and potential. Expert Rev Proteomics. 2016;13:857-71.

3. Kordalewska M, Markuszewski MJ. Metabolomics in cardiovascular diseases. J Pharm Biomed Anal. 2015;113:121-36.

4. Franssens BT, van der Graaf Y, Kappelle LJ, Westerink J, de Borst GJ, Cramer MJ, Visseren FL, Group SS. Body weight, metabolic dysfunction, and risk of type 2 diabetes in patients at high risk for cardiovascular events or with manifest cardiovascular disease: a cohort study. Diabetes Care. 2015;38:1945-51.

5. Omori K, Katakami N, Yamamoto Y, Ninomiya H, Takahara M, Matsuoka TA, Bamba T, Fukusaki E, Shimomura I. Identification of Metabolites Associated with Onset of CAD in Diabetic Patients Using CE-MS Analysis: A Pilot Study. J Atheroscler Thromb. 2019;26:233-45.

6. Ussher JR, Elmariah S, Gerszten RE, Dyck JR. The Emerging Role of Metabolomics in the Diagnosis and Prognosis of Cardiovascular Disease. J Am Coll Cardiol. 2016;68:2850-70.

7. Wang Z, Zhao Y. Gut microbiota derived metabolites in cardiovascular health and disease. Protein Cell. 2018;9:416-31.

8. Dona AC, Coffey S, Figtree G. Translational and emerging clinical applications of metabolomics in cardiovascular disease diagnosis and treatment. Eur J Prev Cardiol. 2016;23:1578-89.

9. Chen ZZ, Gerszten RE. Metabolomics and Proteomics in Type 2 Diabetes. Circ Res. 2020;126:161327.

10. Moller N. Ketone Body, 3-Hydroxybutyrate: Minor Metabolite - Major Medical Manifestations. J Clin Endocrinol Metab. 2020;105.

11. Schugar RC, Moll AR, Andre d'Avignon D, Weinheimer CJ, Kovacs A, Crawford PA. Cardiomyocytespecific deficiency of ketone body metabolism promotes accelerated pathological remodeling. Mol Metab. 2014;3:754-69.

12. Vigili de Kreutzenberg S, Avogaro A. The role of point-of-care 3-hydroxybutyrate testing in patients with type 2 diabetes undergoing coronary angiography. J Endocrinol Invest. 2017;40:627-34.

13. Song JP, Chen L, Chen X, Ren J, Zhang NN, Tirasawasdichai T, Hu ZL, Hua W, Hu YR, Tang HR, Chen HV, Hu SS. Elevated plasma beta-hydroxybutyrate predicts adverse outcomes and disease progression in patients with arrhythmogenic cardiomyopathy. Sci Transl Med. 2020;12.

14. Flores-Guerrero JL, Westenbrink BD, Connelly MA, Otvos JD, Groothof D, Shalaurova I, Garcia E, Navis G, de Boer RA, Bakker SJL, Dullaart RPF. Association of beta-hydroxybutyrate with development of heart failure: Sex differences in a Dutch population cohort. Eur J Clin Invest. 2021;51:e13468. 
15. Laborde CM, Mourino-Alvarez L, Posada-Ayala M, Alvarez-Llamas G, Serranillos-Reus MG, Moreu J, Vivanco F, Padial LR, Barderas MG. Plasma metabolomics reveals a potential panel of biomarkers for early diagnosis in acute coronary syndrome. Metabolomics. 2014;10:414-24.

16. Obokata $M$, Negishi $K$, Sunaga $H$, Ishida $H$, Ito $K$, Ogawa $T$, Iso $T$, Ando $Y$, Kurabayashi $M$. Association Between Circulating Ketone Bodies and Worse Outcomes in Hemodialysis Patients. J Am Heart Assoc. 2017;6.

17. Gensini GG. A more meaningful scoring system for determining the severity of coronary heart disease. Am J Cardiol. 1983;51:606.

18. Tang Y, Wang S, Zhang W, Yang R, Yu X, Wang X, Mu H, Li H, Ji F, Chen W, Dong J. A single-run, rapid polarity switching method for simultaneous quantification of cardiovascular disease-related metabolites using liquid chromatography-tandem mass spectrometry. International Journal of Mass Spectrometry. 2021;461.

19. Chen XF, Chen X, Tang X. Short-chain fatty acid, acylation and cardiovascular diseases. Clin Sci (Lond). 2020;134:657-76.

20. Nielsen R, Moller N, Gormsen LC, Tolbod LP, Hansson NH, Sorensen J, Harms HJ, Frokiaer J, Eiskjaer H, Jespersen NR, Mellemkjaer S, Lassen TR, Pryds K, Botker HE, Wiggers H. Cardiovascular Effects of Treatment With the Ketone Body 3-Hydroxybutyrate in Chronic Heart Failure Patients. Circulation. 2019;139:2129-41.

21. Du Z, Shen A, Huang Y, Su L, Lai W, Wang P, Xie Z, Xie Z, Zeng Q, Ren H, Xu D. 1H-NMR-based metabolic analysis of human serum reveals novel markers of myocardial energy expenditure in heart failure patients. PLoS One. 2014;9:e88102.

22. Mahendran Y, Vangipurapu J, Cederberg H, Stancakova A, Pihlajamaki J, Soininen P, Kangas AJ, Paananen J, Civelek M, Saleem NK, Pajukanta P, Lusis AJ, Bonnycastle LL, Morken MA, Collins FS, Mohlke KL, Boehnke M, Ala-Korpela M, Kuusisto J, Laakso M. Association of ketone body levels with hyperglycemia and type 2 diabetes in 9,398 Finnish men. Diabetes. 2013;62:3618-26.

23. Stryeck S, Gastrager M, Degoricija V, Trbusic M, Potocnjak I, Radulovic B, Pregartner G, Berghold A, Madl T, Frank S. Serum Concentrations of Citrate, Tyrosine, 2- and 3- Hydroxybutyrate are Associated with Increased 3-Month Mortality in Acute Heart Failure Patients. Sci Rep. 2019;9:6743.

24. Arima Y, Izumiya Y, Ishida T, Takashio S, Ishii M, Sueta D, Fujisue K, Sakamoto K, Kaikita K, Tsujita K. Myocardial Ischemia Suppresses Ketone Body Utilization. J Am Coll Cardiol. 2019;73:246-7.

25. Hemal K, Pagidipati NJ, Coles A, Dolor RJ, Mark DB, Pellikka PA, Hoffmann U, Litwin SE, Daubert MA, Shah SH, Ariani K, Bullock-Palmer RP, Martinez B, Lee KL, Douglas PS. Sex Differences in Demographics, Risk Factors, Presentation, and Noninvasive Testing in Stable Outpatients With Suspected Coronary Artery Disease: Insights From the PROMISE Trial. JACC Cardiovasc Imaging. 2016;9:337-46.

26. Lee SE, Sung JM, Andreini D, Al-Mallah MH, Budoff MJ, Cademartiri F, Chinnaiyan K, Choi JH, Chun EJ, Conte E, Gottlieb I, Hadamitzky M, Kim YJ, Lee BK, Leipsic JA, Maffei E, Marques H, de Araujo Goncalves P, Pontone G, Shin S, Stone PH, Samady H, Virmani R, Narula J, Berman DS, Shaw LJ, Bax 
JJ, Lin FY, Min JK, Chang HJ. Sex Differences in Compositional Plaque Volume Progression in Patients With Coronary Artery Disease. JACC Cardiovasc Imaging. 2020;13:2386-96.

27. Cotter DG, Schugar RC, Crawford PA. Ketone body metabolism and cardiovascular disease. Am J Physiol Heart Circ Physiol. 2013;304:H1060-76.

28. Murashige D, Jang C, Neinast M, Edwards JJ, Cowan A, Hyman MC, Rabinowitz JD, Frankel DS, Arany Z. Comprehensive quantification of fuel use by the failing and nonfailing human heart. Science. 2020;370:364-8.

29. Bedi KC, Jr., Snyder NW, Brandimarto J, Aziz M, Mesaros C, Worth AJ, Wang LL, Javaheri A, Blair IA, Margulies KB, Rame JE. Evidence for Intramyocardial Disruption of Lipid Metabolism and Increased Myocardial Ketone Utilization in Advanced Human Heart Failure. Circulation. 2016;133:706-16.

30. Lopaschuk GD, Ussher JR, Folmes CD, Jaswal JS, Stanley WC. Myocardial fatty acid metabolism in health and disease. Physiol Rev. 2010;90:207-58.

31. Turer AT, Stevens RD, Bain JR, Muehlbauer MJ, van der Westhuizen J, Mathew JP, Schwinn DA, Glower DD, Newgard CB, Podgoreanu MV. Metabolomic profiling reveals distinct patterns of myocardial substrate use in humans with coronary artery disease or left ventricular dysfunction during surgical ischemia/reperfusion. Circulation. 2009;119:1736-46.

32. Yurista SR, Chong CR, Badimon JJ, Kelly DP, de Boer RA, Westenbrink BD. Therapeutic Potential of Ketone Bodies for Patients With Cardiovascular Disease: JACC State-of-the-Art Review. J Am Coll Cardiol. 2021;77:1660-9.

33. Jensen NJ, Nilsson M, Ingerslev JS, Olsen DA, Fenger M, Svart M, Moller N, Zander M, Miskowiak KW, Rungby $\mathrm{J}$. Effects of beta-hydroxybutyrate on cognition in patients with type 2 diabetes. Eur $\mathrm{J}$ Endocrinol. 2020;182:233-42.

34. Byrne NJ, Soni S, Takahara S, Ferdaoussi M, Al Batran R, Darwesh AM, Levasseur JL, Beker D, Vos DY, Schmidt MA, Alam AS, Maayah ZH, Schertzer JD, Seubert JM, Ussher JR, Dyck JRB. Chronically Elevating Circulating Ketones Can Reduce Cardiac Inflammation and Blunt the Development of Heart Failure. Circ Heart Fail. 2020;13:e006573.

35. Yu Y, Yu Y, Zhang Y, Zhang Z, An W, Zhao X. Treatment with D-beta-hydroxybutyrate protects heart from ischemia/reperfusion injury in mice. Eur J Pharmacol. 2018;829:121-8.

36. Zhang SJ, Li ZH, Zhang YD, Chen J, Li Y, Wu FQ, Wang W, Cui ZJ, Chen GQ. Ketone Body 3Hydroxybutyrate Ameliorates Atherosclerosis via Receptor Gpr109a-Mediated Calcium Influx. Adv Sci (Weinh). 2021;8:2003410.

\section{Tables}

Table 1. Comparison of baseline characteristics of study population according to serum $\beta$-HDBA tertiles. 


\begin{tabular}{|c|c|c|c|c|}
\hline \multirow[t]{2}{*}{ Characteristic $^{\mathrm{a}}$} & \multicolumn{4}{|c|}{ Tertile of Serum Level of $\beta$-HDBA $(\mu \mathrm{g} / \mathrm{mL})$} \\
\hline & $\overline{\text { LOW }}$ & Intermediate & High & $\begin{array}{l}\text { Trend } P \\
\text { value }\end{array}$ \\
\hline $\mathrm{n}$ & 989 & 992 & 989 & 政 \\
\hline Age, y & $65.0 \pm 10.6$ & $65.7 \pm 10.8$ & $65.9 \pm 11.3$ & 0.058 \\
\hline Male, n (\%) & $638(64.5)$ & $618(62.3)$ & $599(60.6)$ & 0.066 \\
\hline $\mathrm{BMI}, \mathrm{kg} / \mathrm{m} 2$ & $25.7 \pm 3.6$ & $25.9 \pm 3.3$ & $25.5 \pm 3.6$ & 0.097 \\
\hline $\mathrm{SBP}, \mathrm{mmHg}$ & $135.6 \pm 18.0$ & $136.9 \pm 18.5$ & $137.6 \pm 18.9$ & 0.017 \\
\hline $\mathrm{DBP}, \mathrm{mmHg}$ & $78.6 \pm 11.0$ & $78.0 \pm 11.1$ & $78.4 \pm 11.6$ & 0.675 \\
\hline Overweight/obesity, n (\%) & $671(67.8)$ & 711 (71.7) & $636(64.3)$ & 0.738 \\
\hline Hypertension, n (\%) & $659(66.6)$ & $673(67.8)$ & $668(67.5)$ & 0.809 \\
\hline Diabetes, n (\%) & $213(21.5)$ & $430(43.3)$ & 403 (40.7) & $<0.001$ \\
\hline Dyslipidemia, n (\%) & $398(40.2)$ & $474(47.8)$ & $440(44.5)$ & 0.041 \\
\hline History of stroke, n (\%) & $101(10.2)$ & $121(12.2)$ & $97(9.8)$ & 0.665 \\
\hline $\begin{array}{l}\text { Family history of premature CAD, } \mathrm{n} \\
(\%)\end{array}$ & $87(8.8)$ & $69(7.0)$ & $73(7.4)$ & 0.041 \\
\hline \multicolumn{5}{|l|}{ Smoking status, n (\%) } \\
\hline Never & $503(50.9)$ & $522(52.6)$ & $532(53.8)$ & 0.107 \\
\hline Former & $152(15.4)$ & 145 (14.6) & 157 (15.9) & \\
\hline Current & $330(33.4)$ & $316(31.9)$ & $298(30.1)$ & \\
\hline \multicolumn{5}{|l|}{ Statins use, n (\%) } \\
\hline No & $551(55.7)$ & $525(52.9)$ & $575(58.1)$ & 0.727 \\
\hline Take statins intermittently & 108 (10.9) & $71(7.2)$ & $88(8.9)$ & \\
\hline Take statins continuously & $238(24.1)$ & $293(29.5)$ & $223(22.5)$ & \\
\hline \multicolumn{5}{|l|}{ Over 1 year, n (\%) } \\
\hline $\mathrm{FBG}, \mathrm{mmol} / \mathrm{L}$ & $6.4 \pm 2.1$ & $\begin{array}{l}7.0 \pm 2.3 \\
3.9+0.9\end{array}$ & $0.8 \pm 2.3$ & $<0.001$ \\
\hline $\mathrm{TC}, \mathrm{mmol} / \mathrm{L}$ & $3.8 \pm 0.9$ & $14.9 \pm 0.9$ & $12(0.0-17)$ & $<0.001$ \\
\hline $\mathrm{TG}, \mathrm{mmol} / \mathrm{L}$ & $\begin{array}{l}1.2(0.9- \\
1.7)\end{array}$ & $1.4(1.0-1.9)$ & $1.2(0.9-1.7)$ & 0.215 \\
\hline HDL-C, mmol/L & $1.0(0.9-$ & $1.0(0.9-1.2)$ & $1.0(0.9-1.2)$ & 0.866 \\
\hline LDL-C, mmol/L & $2.2(1.7-$ & $2.2(1.7-2.8)$ & $2.3(1.8-2.9)$ & $<0.001$ \\
\hline $\begin{array}{l}\text { Crea, } \mu \mathrm{mol} / \mathrm{L} \\
\mathrm{UA}, \mu \mathrm{mol} / \mathrm{L} \\
\beta-\mathrm{HDBA}(\mu \mathrm{g} / \mathrm{mL})\end{array}$ & $\begin{array}{c}72.0 \pm 16.4 \\
323.0 \pm 84.9 \\
2.9(2.2- \\
3.6)\end{array}$ & $\begin{array}{c}71.4 \pm 16.9 \\
328.9 \pm 85.7 \\
5.8(5.0-6.9)\end{array}$ & $\begin{array}{l}71.6 \pm 18.2 \\
329.0 \pm 93.2 \\
16.9(11.5- \\
32.8)\end{array}$ & $\begin{array}{l}0.610 \\
0.136 \\
<0.001\end{array}$ \\
\hline
\end{tabular}

Not all data were available for all patients.

Abbreviations: $\beta$-HDBA, $\beta$-hydroxybutyrate; CAD, coronary artery disease; BMI, body mass index; SBP, systolic blood pressure; DBP, diastolic blood pressure; FBG, fasting blood glucose; TC, total cholesterol; TG, triglyceride; HDL-C, high density lipoprotein cholesterol; LDL-C, low density lipoprotein cholesterol; Crea, creatinine; UA, uric acid.

a Data are mean \pm SD, median (interquartile range) for continuous variables, or percentage for categorical variables.

Table 2. Spearman's correlation of $\beta$-HDBA with traditional CAD risk factors

\begin{tabular}{lcc}
\hline & Correlation coefficients & $P$ \\
\hline Age & 0.041 & 0.027 \\
BMI & -0.032 & 0.087 \\
SBP & 0.040 & 0.028 \\
DBP & -0.011 & 0.542 \\
FBG & 0.067 & $<0.001$ \\
TC & 0.070 & $<0.001$ \\
TG & 0.019 & 0.338 \\
HDL-C & 0.010 & 0.596 \\
LDL-C & 0.066 & 0.001 \\
Crea & -0.017 & 0.377 \\
UA & 0.021 & 0.260 \\
\hline
\end{tabular}

Abbreviations: CAD, coronary artery disease; BMI, body mass index; SBP, Systolic blood pressure; DBP, Diastolic blood pressure; FBG, fasting blood glucose; TC, total cholesterol; TG, triglyceride; HDL-C, high 
density lipoprotein cholesterol; LDL-C, low density lipoprotein cholesterol; Crea, creatinine; UA, uric acid;

Table 3. Odds ratios (95\% confidence intervals) for the severity of coronary artery lesion according to serum $\beta$-HDBA levels

\begin{tabular}{lcc}
\hline & $P$ value & OR (95\% confidence intervals) \\
\hline Age & $<0.001$ & $1.035(1.023-1.048)$ \\
Gender & $<0.001$ & $0.417(0.308-0.564)$ \\
Smoking status & -- & \\
$\quad$ Never & 0.016 & $1.689(1.103-2.587)$ \\
Former & $<0.001$ & $2.082(1.503-2.883)$ \\
Current & 0.102 & $0.802(0.616-1.045)$ \\
Overweight/obesity & 0.020 & $1.353(1.048-1.746)$ \\
Hypertension & 0.877 & $0.981(0.774-1.245)$ \\
Dyslipidemia & $<0.001$ & $2.570(1.979-3.339)$ \\
Diabetes & 0.241 & $1.266(0.853-1.878)$ \\
History of stroke & 0.011 & $1.504(1.097-2.063)$ \\
Family history of & & \\
premature CAD & 0.032 & $1.151(1.012-1.309)$ \\
$\beta$-HDBA & &
\end{tabular}

Adjusted for age, gender, smoking status, obesity or overweight, hypertension, dyslipidemia, diabetes, stroke and family history of premature CAD.

\section{Figures}




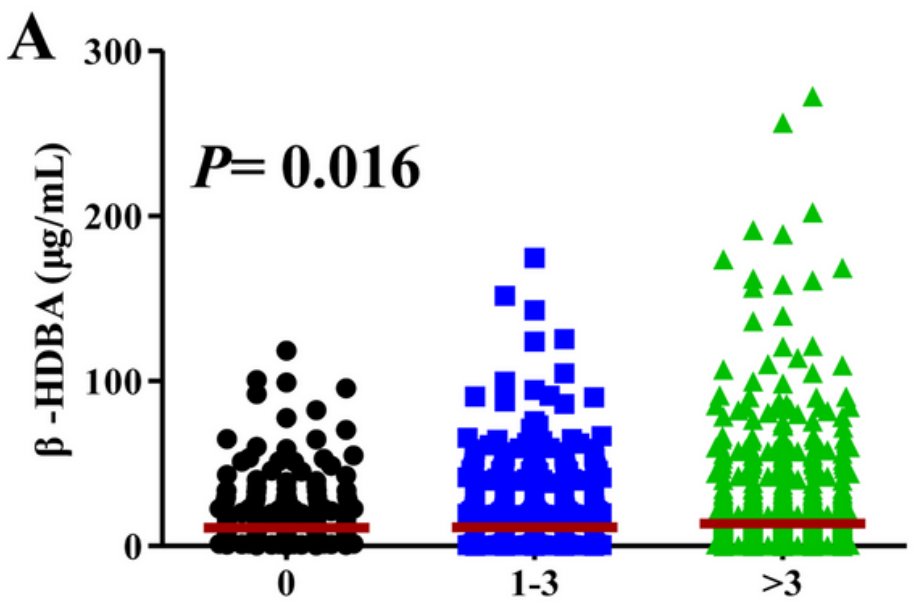

Number of stenosed regions

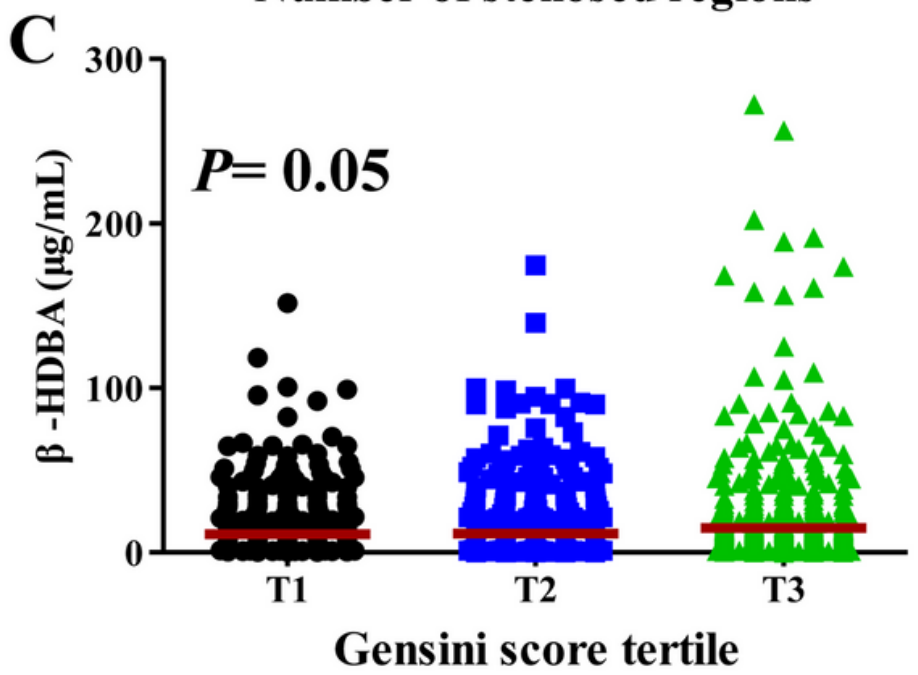

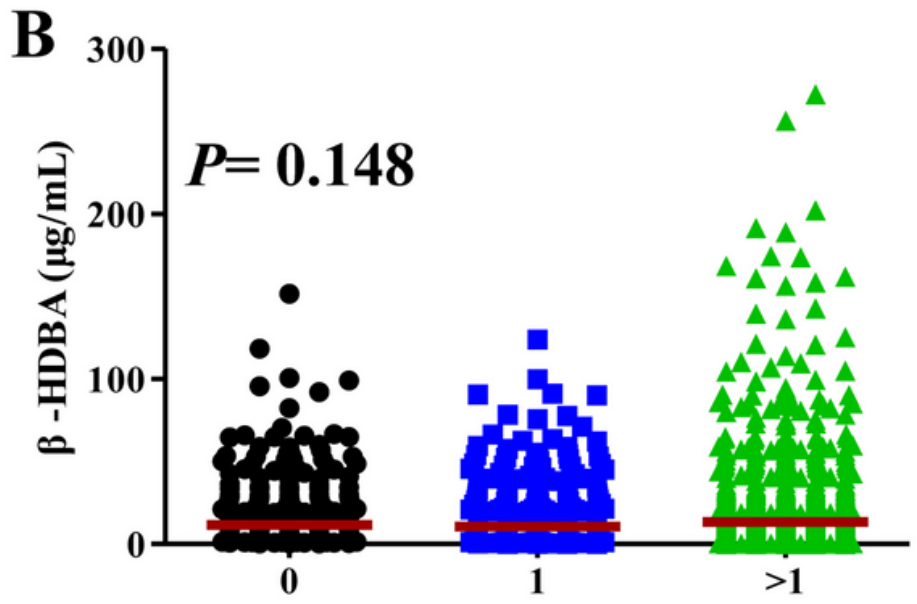

Number of stenosed vessles

Figure 1

Association of serum $\beta$-HDBA with the severity of coronary artery lesion. The association of serum $\beta$ HDBA with CAD severity, according to the number of stenosed regions $(A)$, the number of stenosed vessles $(B)$ and tertile of the Gensini scores $(C) . P<0.05$ considered statistically significant. 


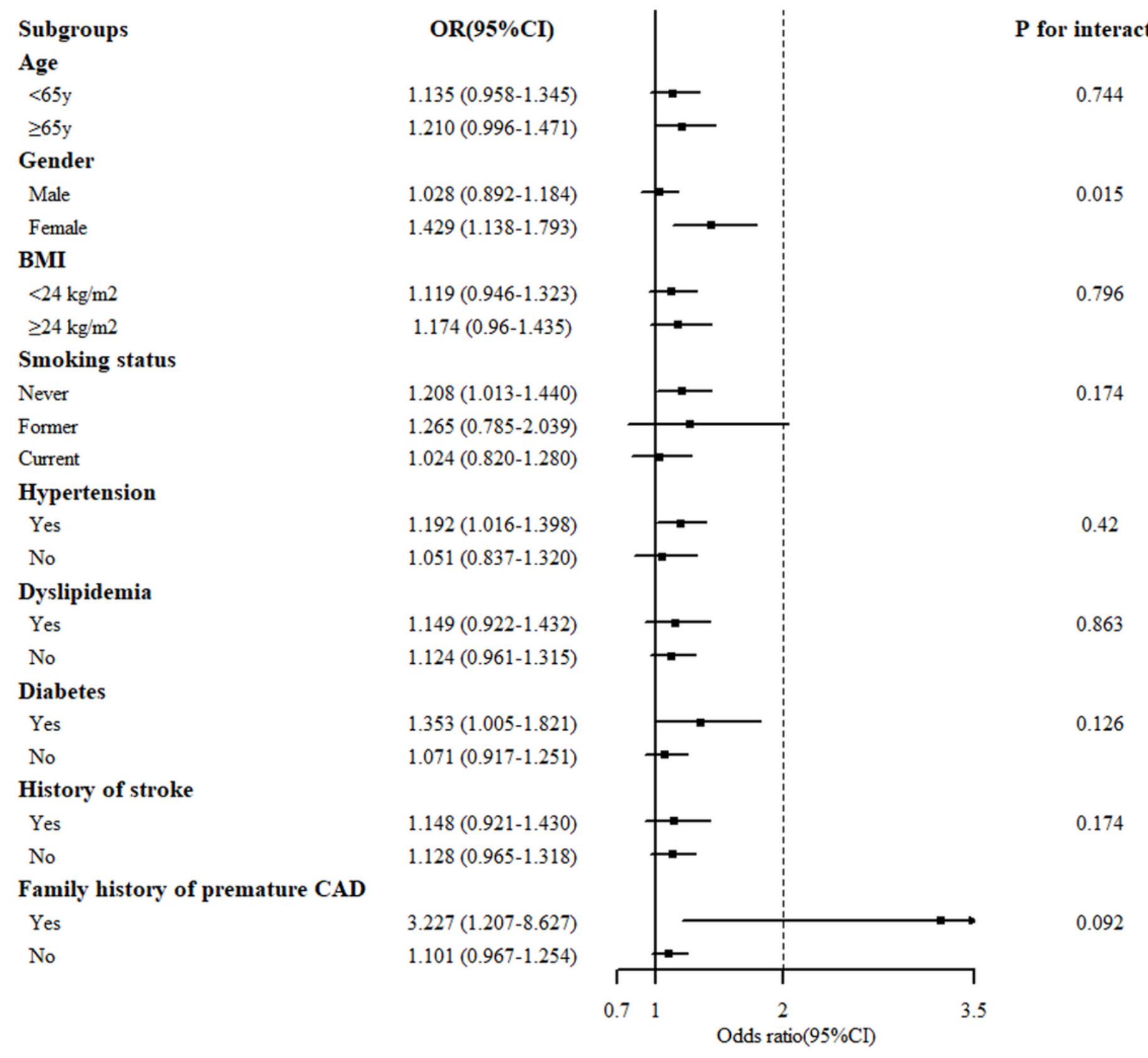

\section{Figure 2}

Stratified analysis of the association [OR $(95 \% \mathrm{Cl})$ ] between circulating $\beta$-HDBA (per 1-SD increment) and the severity of coronary artery lesion. Values are adjusted for age, gender, smoking status, obesity or overweight, hypertension, dyslipidemia, diabetes, stroke and family history of premature CAD, stratifying factors excepted. $P<0.05$ considered statistically significant. 

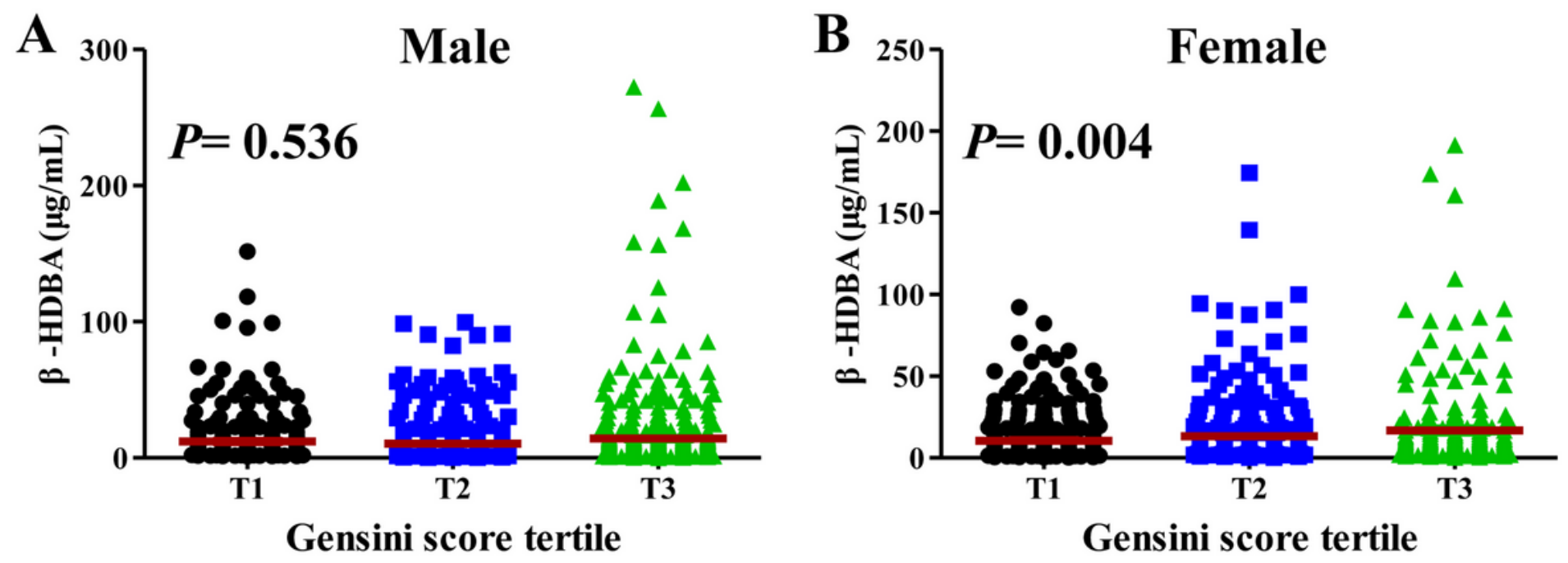

Figure 3

The level of circulating $\beta$-HDBA in male and female respectively according to Gensini score tertile. Serum concentration of $\beta$-HDBA in male (A) and female (B) by Gensini score tertile. $P$ for trend $<0.05$ considered statistically significant.

\section{Supplementary Files}

This is a list of supplementary files associated with this preprint. Click to download.

- Graphicalabstract.tif 